\title{
Polimorfismo genético de interleuquina-1: Asociación con cáncer gástrico en la población de alto riesgo del Centroccidente de Venezuela
}

\author{
Miryan Cañas ${ }^{1 a}$, Yeinmy Morán ${ }^{1 b}$, María Belén Rivero ${ }^{1}$, Adolfo \\ Bohórquez ${ }^{2}$, Venus Villegas' ${ }^{2}$ Yanet Rendón' ${ }^{2}$, Eddy Ramírez ${ }^{2}$, \\ Elvis Valderrama ${ }^{3}$, Zuly Briceño ${ }^{4 c}$, Miguel Angel Chiurillo ${ }^{1}$. \\ Interleukin-1 genetic polymorphism: \\ Association with gastric cancer \\ in the high-risk Central-Western \\ population of Venezuela
}

Background: Genetic predisposition may play a role in the prevalence of gastric cancer (GC). Aim: To investigate the relationship between selected interleukin-1 (IL-1) loci polymorphisms and gastric cancer risk in the Central-Western region of Venezuela, where gastric cancer represents the first cause of cancer-related deaths. Material and methods: In a case-control study, we compared the frequencies of IL-1B-511 and IL-1B+3954 biallelic polymorphism, and the pentallelic VNTR of IL-IRN in 84 gastric adenocarcinoma paraffin-embedded biopsies and 84 endoscopic biopsies from cancer-free controls. Results: No significant increase in genotypic frequencies in gastric cancer was observed for any of the IL-1B-511 allelic combinations. However, in a logistic regression analysis, a significant association emerged for the IL-1B+3954C carrier genotype (odds ratio (OR): 6.2; 95\% confidence intervals (CI) 1.3-28.8). On the other hand, a significantly higher risk was evidenced for the ILIRN $2 / 22$ genotype (OR: 7.0; 95\% CI 2.3-21.5). Only patients with a well/moderately-differentiated adenocarcinoma that were homozygotes for the ILIRN $2 / 2$ genotype, had a higher risk than the complete gastric cancer group (OR: 8.1, 95\% CI 2.5-26.8). Some genotype combinations among IL-1B-511, IL-1B+3954 and IL-IRN showed an increased risk for developing gastric cancer and well/moderate differentiated adenocarcinoma, that was dependent of the presence of ILIRN $2 / 2$ genotype. Conclusions: ILIRN²/2 genotype is associated with increased risk of gastric cancer in the Venezuelan population (Rev Méd Chile 2009; 137: 63-70).

(Key words: Genetic predisposition to disease; Interleukin-1; Stomach neoplasms)

Recibido el 19 de mayo, 2008. Aceptado el 1 de octubre, 2008.

Trabajo financiado por Proyecto 025-ME-2005 CDCHT-UCLA.

${ }^{1}$ Laboratorio de Genética Molecular "Dr. Jorge Yunis-Turbay". Decanato de Ciencias de la Salud, Universidad Centroccidental Lisandro Alvarado (UCLA). Barquisimeto, Venezuela. ${ }^{2}$ Servicio de Gastroenterología, Hospital Antonio María Pineda-UCLA. Barquisimeto, Venezuela. ${ }^{3}$ Departamento de Anatomía Patológica, Hospital Antonio María Pineda-UCLA. Barquisimeto, Venezuela. ${ }^{4}$ Decanato de Ciencia y Tecnología (UCLA). Barquisimeto, Venezuela.

aLicenciado en Biología

${ }^{\mathrm{b}}$ Estudiante de Medicina

'Ingeniero en Informática. MSc en Estadística

Correspondencia a: Dr. Miguel Angel Chiurillo. Laboratorio de Genética Molecular "Dr. Jorge Yunis-Turbay", Decanato de Ciencias de la Salud, Universidad Centroccidental Lisandro Alvarado (UCLA). Barquisimeto. Avenida Libertador. 3001. Estado Lara. Venezuela. Fax: +58-251-259-1886. E mail: mchiurillo@ucla.edu.ve 
$\mathrm{E}^{1}$ cáncer gástrico (CG) es la segunda neoplasia maligna más frecuente en el mundo, solamente superado por el cáncer de pulmón. Aunque la incidencia general ha estado decreciendo en las décadas pasadas, la aparición de adenocarcinoma de la región proximal del estómago y distal del esófago ha presentado un incremento, particularmente en el mundo occidental ${ }^{1}$. En Venezuela el CG representa la segunda causa de muerte por cáncer, sin embargo, en la región centroccidental del país se eleva como la principal causa de mortalidad por cáncer en ambos sexos ${ }^{2}$.

La inflamación crónica de la mucosa del estómago es un prerrequisito para el desarrollo del CG de acuerdo al modelo de múltiples eventos de carcinogénesis gástrica ${ }^{3}$. Por otra parte, la asociación entre la infección por Helicobacter pylori y el riesgo elevado de desarrollar CG está bien establecida en la actualidad ${ }^{3}$. Sin embargo, sólo una pequeña proporción de las personas infectadas por H pylori desarrolla CG; por lo tanto la susceptibilidad genética del huésped debe estar implicada en la evolución de la gastritis. Entre estos factores, se encuentran los genes de citoquinas que participan en la regulación de la respuesta inflamatoria ${ }^{4}$.

El CG avanzado ha sido repetidamente asociado con polimorfismos del grupo de genes de interleuquina-1 (IL-1): IL-1A, IL-1B, e IL-1RN, los cuales codifican para la IL-1 $\alpha$, IL-1ß y el antagonista endógeno del receptor de IL-1 (IL-1ra), respectivamente. Las IL-1 $\alpha$ e IL-1ß actúan como citoquinas proinflamatorias, mientras que IL-1ra se comporta como una citoquina antiinflamatoria que compite con IL-1 $\alpha$ e IL-1ß por la unión a sus receptores. La IL-1ß es importante para el inicio e incremento de la respuesta inflamatoria ante la infección por $\mathrm{H}$ pylori, e igualmente es un poderoso inhibidor de la secreción ácida gástrica. La inhibición prolongada de ésta puede llevar a atrofia de la mucosa de este órgano, la cual es considerada como una lesión precancerosa. Han sido reportados varios polimorfismos bialélicos en los genes de IL-1B, incluyendo IL-1B-511 e IL1B+3954. El gen IL-1RN presenta en el intrón 2 un polimorfismo penta-alélico producto de variaciones en el número de repeticiones en tanda (VNTR) de 86 pares de bases (pb). Los polimorfismos funcionalmente relevantes de estos genes podrían estar correlacionados con variaciones en los niveles de producción de IL-1ß en la respuesta inmune contra $\mathrm{H}$ pylori, y con el desarrollo de alteraciones histológicamente premalignas en la mucosa gástrica 5,6 , así como con un amplio conjunto de condiciones autoinmunes e inflamatorias crónicas ${ }^{6,7}$.

Un estudio pionero en la susceptibilidad a CG demostró que las personas infectadas por $\mathrm{H}$ pylori portadores de los alelos IL-1B-31C o IL-1B-511T, acompañados por el genotipo IL-1RN*2/22, presentaron un incremento de 2 a 3 veces del riesgo de desarrollar $\mathrm{CG}^{5}$. Otros investigadores también mostraron, en diferentes grupos étnicos de todo el mundo, una fuerte asociación entre los polimorfismos proinflamatorios de IL-1 y el incremento del riesgo de desarrollar $\mathrm{CG}^{8-12}$, los cuales también han sido asociados con hipoclorhidria y gastritis atrófica ${ }^{10}$. Sin embargo, varios reportes muestran diferencias en las correlaciones, probablemente debido a la diversidad en el componente genético de las poblaciones humanas ${ }^{12,13}$.

En poblaciones de alto riesgo para CG de Centro y Suramérica también se muestran resultados discordantes. Por ejemplo, los alelos IL1B+3954T e IL-1RN*2 fueron asociados con el riesgo de CG en Costa Rica, pero en individuos del norte de Brasil, no se encontró ninguna asociación entre estos polimorfismos y CG ${ }^{14,15}$. Los reportes contradictorios, así como, las variaciones de acuerdo al origen étnico y geográfico, justifican emprender estudios para evaluar el papel de estos polimorfismos en el desarrollo de CG en diferentes regiones de alta prevalencia. Por ello, en este trabajo se analizaron las frecuencias genotípicas de los polimorfismos de IL-1B-511, IL1B+3954 e IL-1RN para determinar su asociación con el riesgo de desarrollar CG en residentes de la región centroccidental de Venezuela.

\section{PACIENTES Y MÉTOdos}

Muestras. El estudio estuvo conformado por dos grupos de muestras obtenidas de individuos provenientes de la región centroccidental de Venezuela: 1) Grupo control: biopsias de 84 individuos sin evidencias de cáncer gástrico y con diagnóstico histopatológico de gastritis crónica, obtenidas de pacientes del Servicio de Gastroenterología del Hospital Antonio María Pineda (HAMP), Barquisi- 
meto, Venezuela. Se obtuvieron dos biopsias del antro y dos del cuerpo gástrico de cada paciente por endoscopia superior, una de cada región fue destinada a análisis histopatológico y las dos restantes para extracción de ADN. 2) Grupo con CG: 84 biopsias incluidas en parafina de adenocarcinoma gástrico (ADCG) proporcionadas por el Servicio de Anatomía Patológica del HAMP (años 2005-2007).

La edad promedio para los controles fue 58,6 años (18-88 años) y 60,9 años (19-95 años) para los individuos con cáncer gástrico. La relación hombre/mujer fue 48,6/51,4 y 51,1/48,9 para los individuos controles y con cáncer gástrico, respectivamente. Se logró determinar el grado de diferenciación histológica en 78 de las 84 muestras de ADCG, de las cuales 32 (41\%) mostraron poca diferenciación y 46 (59\%) fueron clasificadas como ADCG de moderado o buen grado de diferenciación. Según el tipo histológico, 73 (86,9\%) de las biopsias fueron clasificadas como ADCG de tipo intestinal y 11 (13,1\%) como de tipo difuso.

El estudio reunió los requerimientos éticos del Comité de Bioética del Decanato de Ciencias de la
Salud-UCLA, y todos los participantes dieron su consentimiento informado por escrito.

Aislamiento de ADN. El ADN genómico fue aislado a partir de biopsias incluidas en parafina, previo tratamiento con xileno, mediante el QIAamp tissue Kit (Qiagen) y de biopsias endoscópicas frescas empleando el Wizard Genomic DNA Purification kit (Promega), siguiendo en cada caso las recomendaciones del fabricante.

Asignación de genotipos. La determinación de los polimorfismos fue llevada a cabo por reacción en cadena de la polimerasa y polimorfismos de longitud de fragmentos de restricción (PCR-RFLP) para IL1B-511 e IL-1B+3954, y PCR para IL-1RN, según condiciones descritas previamente ${ }^{16}$, y resumidas en la Tabla 1. Los productos de PCR y sus digestiones fueron analizados mediante electroforesis en geles de agarosa al $2 \%$, teñidos con bromuro de etidio y visualizados con luz UV. Adicionalmente, se hicieron amplificaciones por PCR de un fragmento de $268 \mathrm{pb}$ del gen de ß-globina humana ${ }^{17}$ para asegurar la calidad de las muestras de ADN obtenidas a partir de las biopsias incluidas en parafina.

Tabla 1. Iniciadores y condiciones de PCR empleados para el análisis de los polimorfismos de IL-1B e IL-1RN

\begin{tabular}{|c|c|c|}
\hline Polimorfismo & Iniciadores & $\begin{array}{l}\text { Condiciones de PC R, enzimas de restricción y defini- } \\
\text { ción de los alelos* }\end{array}$ \\
\hline IL-1B-511 & $\begin{array}{l}\text { 511F: 5'-TGGCATTGACTGGTTCAC-3' } \\
\text { 511R: 5'-GTTTAGGAACTTCCCACTT-3' }\end{array}$ & $\begin{array}{l}3 \text { min a } 94^{\circ} \mathrm{C}, 35(40) \text { ciclos de } 45(60) \text { seg a } 94^{\circ} \mathrm{C} \text {, } 45 \\
\text { (90) seg a } 57^{\circ} \mathrm{C}, 30 \text { (90) seg a } 72^{\circ} \mathrm{C} \text {, seguido de } 10 \mathrm{~min} \\
\text { a } 72^{\circ} \mathrm{C} \text {; PCR-RFLP (AvaI); C alelo: } 106 \mathrm{pb} \text { y } 199 \mathrm{pb} \text { T } \\
\text { alelo: } 305 \mathrm{pb} \text {. }\end{array}$ \\
\hline IL-1B+3954 & $\begin{array}{l}\text { 3954F: 5'-GTTGTCACAGACTTTGACC-3' } \\
\text { 3954R: 5'-TTCAGTTCAAGGACCAGA-3' }\end{array}$ & $\begin{array}{l}3 \text { min a } 94^{\circ} \mathrm{C}, 35(40) \text { ciclos de } 45(60) \text { seg a } 94^{\circ} \mathrm{C} \text {, } 45 \\
\text { (90) seg a } 57^{\circ} \mathrm{C}, 30 \text { (90) seg a } 72^{\circ} \mathrm{C} \text {, seguido de } 10 \text { min } \\
\text { a } 72^{\circ} \mathrm{C} \text {; PCR-RFLP (TaqI); C alelo: } 135 \mathrm{pb} \text { y } 114 \mathrm{pb} \text {; } \\
\text { alelo: } 249 \mathrm{pb} \text {. }\end{array}$ \\
\hline IL-1RN & $\begin{array}{l}\text { RNF: 5'-CTCAGCAACACTCCTA-3' } \\
\text { RNR: 5'-TCCTGGTCTGCAGGTAA-3' }\end{array}$ & $\begin{array}{l}3 \text { min a } 94^{\circ} \mathrm{C}, 35(40) \text { ciclos de } 30(60) \text { seg a } 94^{\circ} \mathrm{C}, 30 \\
(90) \text { seg a } 57^{\circ} \mathrm{C}, 20(90) \text { seg a } 72^{\circ} \mathrm{C} \text {, seguido de } 10 \text { min } \\
\text { a } 72^{\circ} \mathrm{C} \text {; PCR; alelo } 1=410 \mathrm{pb} \text { (cuatro repeticiones), alelo } \\
2=240 \mathrm{pb} \text { (dos repeticiones), alelo } 3=500 \mathrm{pb} \text { (cinco } \\
\text { repeticiones), alelo } 4=325 \mathrm{pb} \text { (tres repeticiones), alelo } \\
5=595 \mathrm{pb} \text { (seis repeticiones) }\end{array}$ \\
\hline
\end{tabular}

pb: pares de bases.

*Los tiempos y números de ciclos presentados entre paréntesis fueron empleados para amplificar ADN extraídos a partir de muestras incluidas en parafina. 
Detección de Helicobacter pylori. Para cada muestra se llevaron a cabo dos reacciones de PCR que amplifican blancos diferentes del genoma de $\mathrm{H}$ pylori (H3-H4: 298 pb y Hp1-Hpx2: 150 pb) ${ }^{18}$. El ensayo fue considerado positivo cuando se obtuvo al menos uno de los productos de PCR.

Estadística. Se evaluó el equilibrio Hardy-Weinberg, y el parámetro $\mathrm{D}^{\prime}$ del desequilibrio de ligamiento empleando el programa Arlequin ver. 2.000. Se utilizó la prueba de Chi-cuadrado para comparar las frecuencias genotípicas entre los grupos estudiados. Se calcularon los valores de Odds Ratio (OR) y el 95\% de intervalo de confianza (IC) por regresión logística incondicional con el fin de determinar el riesgo de CG. Para estos dos análisis se utilizó el paquete estadístico SSPS 11.0. Las diferencias fueron consideradas estadísticamente significativas para valores de $\mathrm{P}<0,05$.

\section{Resultados}

Un alto porcentaje de las muestras estudiadas (80\%) con gastritis crónica y CG fueron positivas a $\mathrm{H}$ pylori, no encontrándose diferencia en ese aspecto entre lo grupos analizados. Debido al alto porcentaje de ADCG de tipo intestinal (86,9\%), no se consideró el tipo histológico de las muestras de CG en los análisis estadísticos llevados a cabo.

Polimorfismos de IL-1 y riesgo de cáncer gástrico. La calidad y cantidad de ADN fue insuficiente para evaluar los polimorfismos de IL-1B-511 e IL-1RN en tres muestras de ADCG. Las frecuencias genotípicas de los polimorfismos IL-1B-511T/C, IL1B+3954C/T, e IL-1RN en el grupo control fueron halladas en equilibrio Hardy-Weinberg. No hubo diferencias significativas en la distribución de genotipos de IL-1B-511 entre los grupos analizados (Tabla 2). Sin embargo, para el caso de IL1B+3954, se encontró una asociación significativa con un OR: 6,2 (95\% IC 1,3-28,8) para los portadores del alelo C $(\mathrm{CC}+\mathrm{CT})$ (Tabla 2$)$.

El genotipo IL-1RN*2/2 fue significativamente más frecuente en pacientes con CG $(25,9 \%)$ que en los controles con gastritis crónica $(4,8 \%)$ (Tabla 3). En este caso, el riesgo de desarrollar CG en los portadores del alelo IL-1RN*2 en condición homocigoto se incrementó significativamente (OR: 7,0; 95\% IC 2,3-21,5). Igualmente, se encontró que los portadores del alelo IL-1RN*2 presentaron un incremento ligero del riesgo de desarrollar ADCG con un OR de 2,4 (95\% IC 1,3-4,6).

Cuando se consideró el grado de diferenciación histológica de ADCG, no se encontraron

Tabla 2. Comparación de las frecuencias genotípicas de IL-1B-511 e IL-1B +3954 entre controles e individuos con cáncer gástrico

\begin{tabular}{|lccc|}
\hline Genotipo & $\begin{array}{c}\text { Controles } \\
\mathbf{( n = 8 4 )}\end{array}$ & \multicolumn{2}{c|}{$\begin{array}{c}\text { Cáncer gástrico } \\
\text { (n =84)* }\end{array}$} \\
& $\mathbf{N}(\%)$ & $\mathbf{N}(\%)$ & OR (95\% IC) \\
\hline IL-1B-511 & & $18(22,2)$ & 1,0 \\
C/C (Ref.) & $23(27,4)$ & $47(58,0)$ & $1,7(0,9-3,1)$ \\
C/T & $38(45,2)$ & $16(19,8)$ & $0,7(0,3-1,4)$ \\
T/T & $23(27,4)$ & $63(77,8)$ & $1,3(0,6-2,7)$ \\
Portador de T & $61(72,6)$ & $2(2,4)$ & 1,0 \\
IL-1B+3954 & $11(13,1)$ & $17(20,2)$ & $1,3(0,6-2,8)$ \\
T/T (Ref.) & $14(16,7)$ & $65(77,4)$ & $1,5(0,7-2,9)$ \\
C/T & $59(70,2)$ & $82(97,6)$ & $6,2(1,3-28,8)$ \\
C/C & $73(86,9)$ & & \\
Portador de C & & & \\
\hline
\end{tabular}

OR: odds ratio; Ref: referente.

*El número de muestras fue diferente de acuerdo al polimorfismo analizado. 


\section{Tabla 3. Comparación de las frecuencias genotípicas de IL-1RN entre controles e individuos con cáncer gástrico, casos totales y clasificados de acuerdo al grado de diferenciación histológica del tumor}

\begin{tabular}{|c|c|c|c|c|c|c|c|}
\hline \multirow[t]{2}{*}{ Genotipo } & \multirow{2}{*}{$\begin{array}{l}\text { Controles } \\
(n=84) \\
N(\%)\end{array}$} & \multicolumn{2}{|c|}{$\begin{array}{l}\text { Cáncer gástrico } \\
\qquad(\mathrm{n}=81)^{*}\end{array}$} & \multicolumn{2}{|c|}{$\begin{array}{l}\text { ADCG_Poco diferenciado } \\
(n=30)^{*}\end{array}$} & \multicolumn{2}{|c|}{$\begin{array}{l}\text { ADCG_Moderado/Bien diferenciado } \\
\qquad(n=45)^{*}\end{array}$} \\
\hline & & N (\%) & $\begin{array}{c}\text { OR } \\
(95 \% \text { IC) }\end{array}$ & N (\%) & $\begin{array}{c}\text { OR } \\
(95 \% \text { IC) }\end{array}$ & N (\%) & $\begin{array}{c}\text { OR } \\
(95 \% \mathrm{IC})\end{array}$ \\
\hline \multicolumn{8}{|l|}{ IL-1RN } \\
\hline 1/1 (Ref.) & $43(51,2)$ & $23(28,4)$ & 1,0 & $10(33,3)$ & $0,5(0,2-1,3)$ & $12(26,7)$ & 1,0 \\
\hline $1 / 2$ & $34(40,5)$ & $33(40,7)$ & $1,0(0,5-1,9)$ & $12(40,1)$ & $1,0(0,4-2,3)$ & $18(40,0)$ & $1,0(0,5-2,1)$ \\
\hline $2 / 2$ & $4(4,8)$ & $21(25,9)$ & $7,0(2,3-21,5)$ & $7(23,3)$ & $6,1(1,6-22,6)$ & $13(28,9)$ & $8,1(2,5-26,8)$ \\
\hline $1 / 3$ & $3(3,6)$ & $1(1,2)$ & $0,3(0,0-3,3)$ & & & & \\
\hline \multicolumn{8}{|l|}{$2 / 3$} \\
\hline $1 / 4$ & & $3(3,7)$ & & $1(3,3)$ & $2,9(0,2-47,2)$ & $2(4,4)$ & $3,9(0,3-43,8)$ \\
\hline Portador de 2 & $38(45,2)$ & $54(66,7)$ & $2,4(1,3-4,6)$ & $19(63,3)$ & $2,1(0,9-4,9)$ & $31(68,9)$ & $2,7(1,2-5,8)$ \\
\hline
\end{tabular}

ADCG: adenocarcinoma gástrico; OR: odds ratio; IC: intervalo de confianza; Ref.: referente.

*El número de muestras corresponde a aquellas en las cuales se logró determinar el polimorfismo de IL-1RN.

diferencias significativas en las frecuencias genotípicas de IL-1B-511 e IL-1B+3954. En contraste, sí las hubo para IL-1RN*2/*2, al comparar los controles con los dos grupos de ADCG (poco y moderado/bien diferenciado) (Tabla 3). Sin embargo, solamente en el caso de ADCG moderado/ bien diferenciado el riesgo fue mayor al obtenido con el grupo completo de CG (OR: 8,1; 95\% IC 2,5-26,8) (Tabla 3).

Interacción de polimorfismos de IL-1B e IL-1RN. No se observó un fuerte desequilibrio de ligamiento entre los alelos de los polimorfismos evaluados. La frecuencia del genotipo combinado IL-1B+3954C/C-IL$1 \mathrm{RN} * 2 / * 2$ fue significativamente mayor en los grupos con CG $(23,5 \%)$ y ADCG moderado/bien diferenciado $(28,9 \%)$ que en los controles $(3,6 \%)$. Esto corresponde a un incremento del niesgo de 8,3 y 11 veces para CG y ADCG moderado/bien diferenciado, respectivamente (Tabla 4). Sin embargo, en los modelos de regresión logística, sólo el término para el genotipo IL-1RN*2/*2 fue significativo.

Por otra parte, el genotipo considerado como proinflamatorio IL-1B-511T fue encontrado en dos combinaciones que presentaron variaciones estadísticamente significativas de las frecuencias entre grupos (Tabla 4). Particularmente, el genotipo combinado IL-1B-511T-IL-1B+3954C-IL-1RN*2/*2 originó un incremento del riesgo para CG y ADCG moderado/bien diferenciado de 10,6 (95\% IC 2,3$47,5)$ y $13,7(95 \%$ IC $2,9-65,0)$ veces, respectivamente. El riesgo conferido por el polimorfismo IL-1B-511 fue también dependiente de la interacción con el genotipo IL-1RN*2/*2.

\section{DisCUSIÓN}

El cáncer gástrico es el resultado de la interacción entre factores genéticos del hospedero con factores ambientales como la dieta y la infección por $\mathrm{H}$ pylori. Los polimorfismos genéticos del loci IL-1 han sido adicionados como una de las últimas piezas en el proceso de la carcinogénesis gástrica, por lo tanto el análisis de estos polimorfismos puede ser empleado como una herramienta en la búsqueda de variaciones en la susceptibilidad genética individual en procesos relevantes para la aparición del cáncer gástrico, cuyos mecanismos son todavía relativamente desconocidos.

En este trabajo, el alelo IL-1RN*2 se encontró asociado con un incremento significativo del riesgo de CG en la población del centroccidente venezolano, siendo esta asociación aún mayor con el genotipo homocigoto IL-1RN*2/*2 (OR: 7,0; 95\% IC 2,3-21,5). La asociación del genotipo IL$1 \mathrm{RN} * 2 / 2$ con CG fue descrita inicialmente por ElOmar y $\mathrm{Col}^{5}$, y posteriormente se ha 
Tabla 4. Comparación entre las frecuencias de genotipos combinados de IL-1B e IL-1RN en controles e individuos con cáncer gástrico y adenocarcinoma gástrico moderado/bien diferenciado

\begin{tabular}{|c|c|c|c|c|c|c|c|}
\hline \multirow[b]{2}{*}{ IL-1B-511 } & \multicolumn{2}{|c|}{ Genotipos combinados } & \multirow{2}{*}{$\begin{array}{c}\text { Controles } \\
\text { (\%) }\end{array}$} & \multicolumn{2}{|c|}{ C áncer gástrico } & \multicolumn{2}{|c|}{$\begin{array}{l}\text { ADCG_Moderado/ } \\
\text { Bien diferenciado }\end{array}$} \\
\hline & IL-1B+3954 & IL-IRN & & $(\%)$ & OR $(95 \%$ IC $)$ & $(\%)$ OR & $(95 \%$ IC $)$ \\
\hline \multirow{3}{*}{ Portador de T } & $\mathrm{C} / \mathrm{C}$ & 1/1 (Ref.) & 35,7 & 19,8 & 1,0 & 15,6 & \\
\hline & & $2 / 2$ & 3,6 & 20,5 & $7,0 \quad(1,9-25,0)$ & $25,0 \quad 9,0$ & $(2,4-34,3)$ \\
\hline & $\mathrm{C} / \mathrm{C}$ & $2 / 2$ & 3,6 & 23,5 & $8,3 \quad(2,3-29,2)$ & $28,9 \quad 11,0$ & $(2,9-41,1)$ \\
\hline Portador de $\mathrm{T}$ & Portador de C & $2 / 2$ & 2,4 & 20,5 & $10,6 \quad(2,3-47,5)$ & $25,0 \quad 13,7$ & $(2,9-65,0)$ \\
\hline
\end{tabular}

ADCG: adenocarcinoma gástrico; OR: odds ratio; IC: intervalo de confianza; Ref.: referente.

correlacionado con el incremento del riesgo de CG en individuos caucásicos pero no en asiáticos, donde dicho alelo es raro ${ }^{13}$. Recientemente esta asociación ha sido confirmada en poblaciones europeas $^{19}$. Igualmente, los resultados de este estudio sugieren que los individuos que poseen el genotipo homocigoto para el alelo IL-1RN*2 presentan un mayor riesgo de desarrollar ADCG con un mejor grado de diferenciación, incluso superior al riesgo de sufrir CG en general (OR: 8,1 vs 7,0).

La región VNTR del IL-1RN contiene tres potenciales sitios de unión a proteínas lo que sugiere una posible relevancia funcional, aun cuando el mecanismo implicado en la asociación entre el alelo IL-1RN*2 y el incremento de la expresión de la IL-1ß sea aún desconocido ${ }^{10}$. La expresión y concentración de IL-1ra en la mucosa parece depender del número de alelos IL-1RN*2, ya que en diferentes formas de colitis los portadores del genotipo homocigoto IL-1RN*2/*2 presen$\tan$ niveles reducidos de IL-1ra ${ }^{20}$. Estudios recientes han asociado los alelos IL-1RN*2 e IL-1B$511 \mathrm{~T}$ con un incremento en la expresión de la Il1ß, y con escasa secreción ácida, inflamación gástrica y carcinoma gástrico en respuesta a la infección por $\mathrm{H}$ pylori $4-6,8,10$. Asimismo, no están claras las razones por las que el alelo IL-1RN*2 pudiera estar más asociado con ADCG moderado/ bien diferenciado, que con tipos poco diferenciados, siendo posible que estén interactuando otros factores para generar esta asociación. Sin embargo, se puede sugerir que el polimorfismo de IL1RN está involucrado en la sobrevivencia, actuando como un factor pronóstico, así como en la susceptibilidad al CG.
La acción proinflamatoria y la asociación con el riesgo para CG del polimorfismo IL-1B+3954 no ha sido tan clara como la que se ha descrito para el alelo IL-1RN*2, aunque algunos reportes han mostrado frecuencias del alelo IL-1B+3954T o del genotipo heterocigoto IL-1B+3954C/T significativamente más elevadas en casos de $\mathrm{CG}^{14,21,22}$. Sin embargo, Wang y $\mathrm{col}^{13}$, en un metaanálisis, mostraron en sus conclusiones que el genotipo IL1B+3954T no está asociado con un incremento del riesgo de desarrollar CG.

La presencia concomitante de los genotipos homocigotos para IL-1B+3954C e IL-1RN*2, fue correlacionada con un riesgo significativo para desarrollar CG, y aun mayor para el subgrupo de ADCG moderado/bien diferenciado. Adicionalmente, la asociación de los genotipos portador del alelo IL-1B-511T e IL-1RN*2/22 causó un incremento del riesgo para CG y ADCG moderado/ bien diferenciado. De manera similar, Ruzzo y $\mathrm{Col}^{23}$, reportan que cuando el alelo IL-1B-31C fue analizado individualmente no estuvo asociado con la enfermedad. Sin embargo, en combinación con alelos de IL-1B-511 e IL-1RN, resultó incluido en un haplotipo de alto riesgo para el desarrollo de CG de tipo intestinal. Adicionalmente, El-Omar y $\mathrm{Col}^{9}$, confirmaron que la presencia de múltiples polimorfismos proinflamatorios confiere un mayor riesgo para el desarrollo de CG distal. Según los resultados de este trabajo, sería posible que exista una relación funcional sinergística de los tres polimorfismos en desórdenes gástricos, como ha sido observada en portadores del genotipo IL-1B$511 \mathrm{~T} / \mathrm{T}$ y el alelo IL-1RN*2 con niveles incrementados de la IL-1ß en la mucosa gástrica ${ }^{6,10}$. 
En este trabajo no se encontró correlación entre la presencia de infección por $\mathrm{H}$ pylori y alguno de los polimorfismos estudiados. Venezuela presenta tasas de infección por esta bacteria extremadamente elevadas $(>90 \%)^{24}$, y aunque algunos estudios han sido conducidos en lesiones precancerosas ${ }^{25,26}$, es necesario completar más investigaciones sobre el papel de los factores genéticos del huésped involucrados en determinar la evolución de la gastritis a cáncer en este país.

En conclusión, nuestro estudio en esta población de Venezuela provee evidencias de que el genotipo homocigoto de IL-1RN*2 puede jugar un papel en la etiología del CG, siendo aún mayor la

\section{REFERENCIAS}

1. Parkin M, Bray F, Devesa S. Cancer burden in the year 2000. The global picture. Europ J Cancer 2001; 37: S4-S66.

2. Ministerio del Poder Popular para la Salud. República Bolivariana de Venezuela. Anuario de Mortalidad 2005. http://www.mpps.gob.ve; 2006.

3. Fox JG, Wang TC. Inflammation, atrophy, and gastric cancer. J Clin Invest 2007; 117: 60-9.

4. Rad R, Dossumbekova A, Neu B, Lang R, Bauer S, Saur $D$ ET AL. Cytokine gene polymorphisms influence mucosal cytokine expression, gastric inflammation, and host specific colonization during Helicobacter pylori infection. Gut 2004; 53: 1082-9.

5. El-Omar EM, Carrington M, Chow WH, Mccoll KE, BrEAm JH, Young HA ET AL. Interleukin-1 polymorphisms associated with increased risk of gastric cancer. Nature 2000; 404: 398-402.

6. Hwang IR, Kodama T, Kikuchi S, Sakai K, Peterson LE, Graham DY ET aL. Effect of interleukin 1 polymorphisms on gastric mucosal interleukin $1 \mathrm{~b}$ production in Helicobacter pylori infection. Gastroenterology 2002; 123: 1793-803.

7. AREND WP. The balance between IL-1 and IL-1Ra in disease. Cytokine Growth Factor Rev 2002; 13: 32340.

8. Machado JC, Pharoah P, Sousa S, Carvalho R, Oluveira C, Figueiredo $\mathrm{C}$ ET AL. Interleukin $1 \mathrm{~B}$ and interleukin $1 \mathrm{RN}$ polymorphisms are associated with increased risk of gastric carcinoma. Gastroenterology 2001; 121: 823-9.

9. El-Omar EM, Rabin CS, Gammon MD, Vaughan TL, Risch HA, Schoenberg JB ET AL. Increased risk of noncardia gastric cancer associated with proinflammatory cytokine gene polymorphisms. Gastroenterology 2003; 124: 1193-201. contribución de este polimorfismo al desarrollo del ADCG moderado/bien diferenciado. La asociación potencial de la combinación alélica IL-1B511T-(IL-1B+3954C)-IL-1RN*2 con el desarrollo de CG no es descartable. Por otra parte, son necesarios trabajos adicionales con un mayor número de muestras e implicando otras variables para profundizar en el conocimiento sobre la interacciones entre genes y el ambiente en la susceptibilidad al CG.

Agradecimientos

A Lolymar Mendoza y María Eugenia Camargo por su asistencia técnica.

10. Furuta T, Shirai N, Sugimoto M. Controversy in polymorphisms of interleukin-1beta in gastric cancer risks. J Gastroenterol 2004; 39: 501-3.

11. Palu D, Saieva C, Luzzi I, Masala G, Topa S, Sera F et AL. Interleukin-1 gene polymorphisms and gastric cancer risk in a high-risk Italian population. Am J Gastroenterol 2005; 100: 1941-8.

12. Pérez-Pérez GI, Garza-González E, Portal C, Olivares AZ. Role of cytokine polymorphisms in the risk of distal gastric cancer development. Cancer Epidemiol. Biomarkers Prev 2005; 14: 1869-73.

13. Wang P, Xia HHX, Zhang JY, Dai LP, Xu XQ, Wang KJ. Association of interleukin-1 gene polymorphisms with gastric cancer: A meta-analysis. Int J Cancer 2006; 120: 552-62.

14. Alpizar-Alpizar W, Pérez-Pérez Gi, Une C, Cuenca P, SIERRA R. Association of interleukin-1B and interleukin-1RN polymorphisms with gastric cancer in a high-risk population of Costa Rica. Clin Exp Med 2005; 5: 169-76.

15. Gatti LL, Burbano RR, De AssumpÇão PP, Smith M de A, PAỸ̃o SL. Interleukin-1ß polymorphisms, Helicobacter pylori infection in individuals from Northern Brazil with gastric adenocarcinoma. Clin Exp Med 2004; 4: 93-8.

16. Nemetz A, Tóth M, García-GonzÁlez MA, ZÁgoni T, FEHÉR J, PEÑA AS ET AL Allelic variation at the interleukin 1ß gene is associated with decreased bone mass in patients with inflammatory bowel diseases. Gut 2001; 49: 644-9.

17. Saiki RK, Scharf S, Faloona F, Mulus KB, Horn GT, ERLCH HA ET AL Enzymatic amplification of betaglobin genomic sequences and restriction site analysis for diagnosis of sickle cell anemia. Science 1985; 230: 1350-4.

18. Gatti LL, De Lábio R, Da Silva LC, Smithand M De A, PaYão SL cagA Positive Helicobacter pylori in Brazi- 
lian Children Related to Chronic Gastritis. Braz J Infect Dis 2006; 10: 254-8.

19. Crusius JBa, Canzian F, Capeliá G, Peña AS, Pera G, Sala $\mathrm{N}$ ET AL. Cytokine gene polymorphisms and the risk of adenocarcinoma of the stomach in the European prospective investigation into cancer and nutrition (EPIC-EURGAST). Ann Oncol 2008; 19: 1894-902.

20. Andus T, Daig R, Vogl D, Aschenbrenner E, Lock G, HoLeRBACH S ET AL. Imbalance of the interleukin 1 system in colonic mucosa: Association with intestinal inflammation and interleukin 1 receptor antagonist genotype 2. Gut 1997; 41: 651-7.

21. Sakuma K, Uozaki H, Chong JM, Hironaka M, Sudo M, UshiKU T ET AL. Cancer risk to the gastric corpus in Japanese, its correlation with interleukin-1b gene polymorphism 13953*T. and Epstein-Barr virus infection. Int J Cancer 2005; 115: 93-7.

22. Zhang WH, Wang XL, Zhou J, An LZ, Xie XD. Association of interleukin-1B (IL-1B) gene polymorphisms with risk of gastric in Chinese population. Cytokine 2005; 30: 378-81.
23. Ruzzo A, Graziano F, Pizzagalu F, Santini D, Battistem $\mathrm{V}$, PANUNZI S ET AL. Interleukin 1B gene IL-1B and interleukin 1 receptor antagonist gene IL-1RN polymorphisms in Helicobacter pylori negative gastric cancer of intestinal and diffuse histotype. Ann Oncol 2005; 16: 887-92.

24. Ghose C, Pérez-Pérez GI, Van Doorn LJ, DomínguezBelLo MG, Blaser MJ. High frequency of gastric colonization with multiple Helicobacter pylori strains in Venezuelan subjects. J Clin Microbiol 2005; 43: 2635-41.

25. Kato I, Canzian F, Franceschi S, Plummer M, Van Doorn LJ, LU Y ET AL. Genetic polymorphisms in antiinflamatory cytokine signaling and the prevalence of gastric precancerous lesions in Venezuela. Cancer Causes Control 2006; 17: 1183-91.

26. Kato I, Van Doorn LJ, Canzian F, Plummer M, FranCESCHI S, Vivas J ET aL. Host-bacterial interaction in the development of gastric precancerous lesions in a high risk population for gastric cancer in Venezuela. Int J Cancer 2006; 119: 1666-71. 